\title{
Resolving the 3D rotational and translational dynamics of single molecules using radially and azimuthally polarized fluorescence
}

\author{
Oumeng Zhang, ${ }^{\dagger}$ Weiyan $\mathrm{Zhou}^{\dagger}{ }^{\dagger}$ Jin $\mathrm{Lu},{ }^{\dagger}$ Tingting $\mathrm{Wu}^{\dagger}{ }^{\dagger}$ and Matthew D. Lew ${ }^{*, \dagger, \dagger, \uparrow}$ \\ $\dagger$ Department of Electrical and Systems Engineering \\ $\ddagger$ Center for the Science and Engineering of Living Systems \\ IInstitute of Materials Science and Engineering, Washington University in St. Louis, Missouri 63130, USA \\ E-mail: mdlew@wustl.edu
}

\begin{abstract}
We report a radially and azimuthally polarized ( $\mathrm{raPol}$ ) microscope for high detection and estimation performance in single-molecule orientation-localization microscopy (SMOLM). With 5000 photons detected from Nile red (NR) transiently bound within supported lipid bilayers (SLBs), raPol SMOLM achieves $2.9 \mathrm{~nm}$ localization precision, $1.5^{\circ}$ orientation precision, and $0.17 \mathrm{sr}$ precision in estimating rotational wobble. Within DPPC SLBs, SMOLM imaging reveals the existence of randomly oriented binding pockets that prevent NR from freely exploring all orientations. Treating the SLBs with cholesterol-loaded methyl$\beta$-cyclodextrin (M $\beta C D$-chol) causes NR's orientational diffusion to be dramatically reduced, but curiously, NR's median lateral displacements drastically increase from 20.8 $\mathrm{nm}$ to $75.5 \mathrm{~nm}$ (200 ms time lag). These jump diffusion events overwhelmingly originate from cholesterol-rich nanodomains within the SLB. These detailed measurements of single-molecule rotational and translational dynamics are made possible by raPol's high measurement precision and are not detectable in standard SMLM.
\end{abstract}

\section{Keywords}

Single-molecule tracking, phospholipid bilayer, cholesterol condensing, rotational diffusion, lateral diffusion

Beyond improving the localization accuracy 1106 of singlemolecule localization microscopy (SMLM) ${ }^{7 / 10}$ simultaneously imaging molecular positions and orientations in singlemolecule orientation localization microscopy (SMOLM) provides unparalleled insights into biochemical processes. Recent developments in SMOLM allow scientists to resolve the organization of amyloid aggregates, $\frac{11][1]}{13}$ conformations of DNA strands, $\frac{6114}{18}$ and the structure of actin networks. $19 \sqrt{21}$ Using point spread function (PSF) engineering, microscopists have developed various techniques $\frac{41620122123}{\text { to }}$ improve measurement precision, approaching the fundamental performance limits for measuring both orientation $24 \sqrt{26}$ and localization. $[27[28]$

However, many SMOLM techniques exhibit poor measurement precision for molecules that are oriented out of the coverslip plane (e.g., the $x$ - and $y$-polarized ( $x y \mathrm{Pol}$ ) standard PSF $\left.{ }^{12 \mid 29}\right)$. Others improve measurement precision by expanding the imaging system PSF significantly and thus suffer degraded localization precision and detection sensitivity for dim emitters (e.g., the Tri-spot PSF ${ }^{\sqrt{22}}$ ). Despite the recent developments of $\mathrm{CHIDO}^{20}$ and the vortex $\mathrm{PSF}^{\sqrt{6}}$ for imaging the 3D positions and orientations of single molecules (SMs), no existing techniques exhibit sufficiently high localization precision, orientation precision, and sensitivity to SM wobble for simultaneous 3D orientation, wobble, and position tracking of SMs within lipid membranes. Inspired by symmetries within the dipole radiation pattern, we show the first experimental demonstration of radially and azimuthally polarized (raPol) fluorescence, ${ }^{24|26| 30} \mathrm{a}$ variant of the $\mathrm{y}$-Phi PSF ${ }^{\sqrt{5}}$ for highly sensitive position and orientation tracking of SMs. It is implemented using a commercially available vortex (half) wave plate (VWP) and polarizing beamsplitter (PBS) within a widefield epifluorescence microscope. The raPol microscope exhibits excellent orientation estimation performance while maintaining high detection rates and localization performance comparable to those of standard polarized microscopes. We utilize the raPol PSF to study the dynamics of Nile red (NR) molecules transiently bound to supported lipid bilayers (SLBs) ${ }^{[31}$ Imaging NR dynamics within DPPC SLBs reveals the existence of randomly oriented binding pockets that prevent NR from freely rotating. In addition, raPol is capable of tracking simultaneously the position and orientation of NR as it explores SLBs modified by methyl- $\beta$-cyclodextrin loaded with cholesterol (M $\beta C D-$ chol). As cholesterol (chol) is deposited, NR's orientation tilts mostly perpendicular to the SLB, and its rotational diffusion is greatly reduced, but its translational diffusion dramatically increases almost four-fold. These data suggest that NR "jumps" between cholesterol-rich nanodomains within the SLB. To our knowledge, these experiments are the first measurements of how $\mathrm{M} \beta \mathrm{CD}$-chol affects the nanoscale chemical environments within lipid membranes at the singlemolecule level.

We begin by describing a rotationally diffusing molecule using its average orientation $\boldsymbol{\mu}=\left[\mu_{x}, \mu_{y}, \mu_{z}\right]^{\dagger}=$ $[\sin \theta \cos \phi, \sin \theta \sin \phi, \cos \theta]^{\dagger}$ within a hard-edged cone of solid angle $\Omega$ (Figure 17 ), where $\mu_{z}$ is parallel to the optical axis, $\Omega=0$ represents a rotationally-fixed molecule, and $\Omega=2 \pi$ represents a freely-wobbling molecule. Here, we assume that the SM wobbles uniformly within the cone for simplicity, and our analysis may be easily adapted for other rotational potential wells or geometries. $.32 \mid 34$

Due to the symmetry of the dipole emission pattern, the photons emitted by an SM oriented parallel to the optical axis $\left(\mu_{z}=1\right)$ captured by an objective lens are radially polarized. $\sqrt[3556]{36}$ Therefore, an intuitive way to distinguish outof-plane molecules from in-plane ones is to measure radially vs. azimuthally polarized fluorescence. We achieve this po- 
larization separation by adding a VWP (Figure 1p) to the back focal plane (BFP) of a microscope with two polarized imaging channels. The spatially-varying fast axis direction of the VWP turns the radially and azimuthally polarized light to $x$ and $y$-polarized light. A PBS is then used to separate the fluorescence into radially and azimuthally polarized images (Figure S1 1). As a molecule rotates out of plane, i.e., as $\mu_{z}$ increases, photons shift from the azimuthally polarized to the radially polarized channel. When the SM is aligned with the optical axis, all fluorescence photons are confined to the radially polarized image (Figure 1, red).

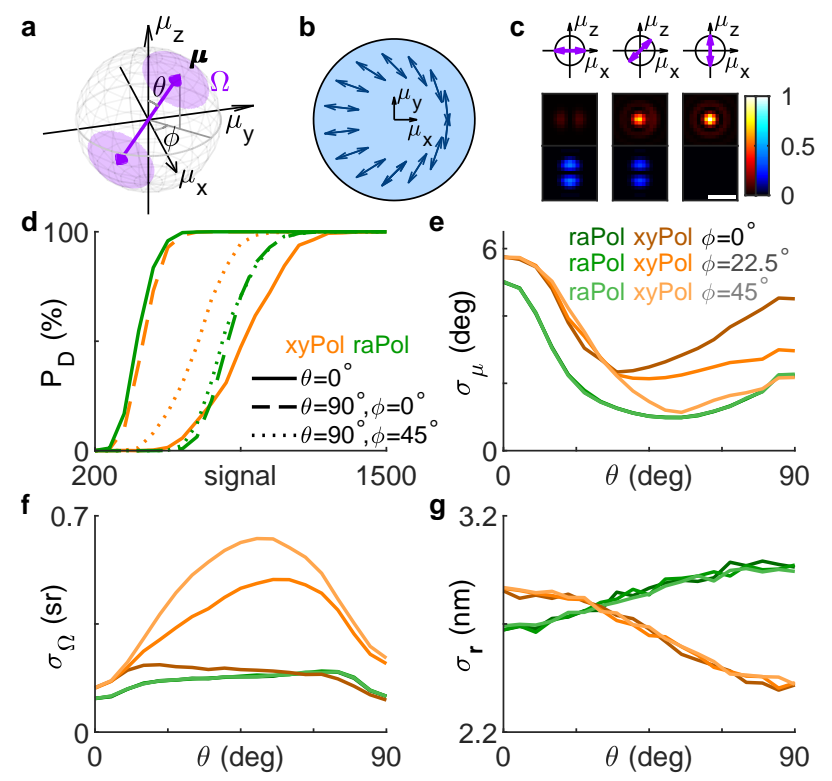

Figure 1: The radially and azimuthally polarized (raPol) standard PSF. (a) The rotational diffusion of a fluorescent molecule is described using a unit vector $\boldsymbol{\mu}$ and a wobble solid angle $\Omega$ on the surface of a hard-edged cone. (b) A vortex (half) waveplate (VWP) is placed at the back focal plane of the imaging system, transforming radially and azimuthally polarized light to $x$ and $y$-polarized light, respectively. Arrows represent the VWP's fast axis. (c) Representative radially (red) and azimuthally (blue) polarized PSFs of rotationally-fixed molecules with polar angles $\theta$ of $90^{\circ}, 45^{\circ}$, and $0^{\circ}$. Colorbar: normalized intensity. Scale bar: $500 \mathrm{~nm}$. (d-g) Detection and estimation performance of a sparsity-promoting maximum-likelihood estimation algorithm using simulated $x y \mathrm{Pol}$ (orange) and raPol (green) images. (d) Detection rate $P_{\mathrm{D}}$ as a function of the number of total signal photons detected from an SM with various orientations and a fixed wobble $\Omega=0.28 \pi$ sr. Dashed, solid, and dotted lines represent molecular orientations $\theta=0^{\circ}$, $[\theta, \phi]=\left[90^{\circ}, 0^{\circ}\right]$, and $[\theta, \phi]=\left[90^{\circ}, 45^{\circ}\right]$, respectively. g) Precision of estimating the (e) average orientation $\boldsymbol{\mu}$, (f) wobble angle $\Omega$, and (g) 2D position $\boldsymbol{r}$ of an SM with 5000 signal photons detected for various polar angles $\theta$. Dark, medium, and light colors represent azimuthal angles $\phi=0^{\circ}$, $\phi=40^{\circ}$, and $\phi=90^{\circ}$, respectively. The background is 30 photons per camera pixel $\left(66.86 \times 66.86 \mathrm{~nm}^{2}\right)$.

High detection rates are critical for collecting unbiased measurements of SM dynamics. We quantify the detection rate $P_{\mathrm{D}}$ of raPol compared to $x y$ Pol, a common easy-toimplement SMOLM technique, using RoSE-O, a sparsitypromoting joint detection and maximum-likelihood estima- tion algorithm. ${ }^{18}$ We find that despite the orientation dependence, the detection performance of raPol is comparable to that of $x y \mathrm{Pol}$ (Figure $1 \mathrm{~d}$ and Figure S3). Thus, dim SMs are detected more reliably using raPol as opposed to most engineered PSFs optimized for SMOLM; it is more difficult to detect weak emitters using larger PSFs [Figure S3.(iii,iv)]. Due to the toroidal, polarized emission pattern of an SM, the detection rate using $\mathrm{raPol}$ is much better than that of $x y \mathrm{Pol}$ for $z$-oriented molecules since the energy is concentrated in the radially polarized channel; with 500 signal photons detected, raPol achieves a detection rate of $92.5 \%$ while $x y \mathrm{Pol}$ can only detect less than $1 \%$ of the SMs (Figure 11). The superior detection rate for this orientation motivates us to adopt raPol for lipid membrane imaging (see below). However, its detection rate is worse than that of $x y$ Pol for inplane molecules for the opposite reason (Figure 1 estingly, due to the symmetry of raPol, its detection performance is not affected by the azimuthal angle $\phi$, whereas that of $x y \mathrm{Pol}$ may vary up to a factor of 6 across various $\phi$ (Figure S3, b).

Next, we compare the orientation and position measurement precision for raPol and $x y \mathrm{Pol}$, evaluated at an SBR such that we achieve $100 \%$ detection rate using both PSFs. The precision of measuring the average orientation $\sigma_{\mu}$ is quantified as the angle subtended by an arc that represents measurement uncertainty on the orientation unit sphere (SI Section 2.2. Strikingly, raPol exhibits superior orientation and wobble measurement precision over $x y$ Pol for almost all possible SM orientations [Figure 1 1 , f and Figure S5a,b(ii,v)]. The average $2 \mathrm{D}$ localization precision $\sigma_{r}$ using raPol is only $12 \%$ worse compared to that of $x y \mathrm{Pol}$ [Figure 18 and Figure $[\mathrm{S} 5 \mathrm{a}, \mathrm{b}(\mathrm{i})]$, where $x y \mathrm{Pol}$ is usually perceived to be optimal for localizing in-focus molecules. Further Cramér-Rao bound (CRB, SI Section 2.2 $\frac{37}{3}$ analysis shows that the orientation performance of raPol is comparable to recently developed engineered PSFs while exhibiting a much better localization precision (Figure 44 and Table S1).

While the translational dynamics of fluorophore-lipid interactions have been characterized at the SM level, e.g., by fluorescence correlation spectroscopy (FCS), $\stackrel{41}{\text { relatively }}$ little is known about their rotational dynamics. We next study the rotational dynamics of Nile red (Figure 2 a) within $\mathrm{SLBs}^{31}$ (Figure 20 using points accumulation for imaging in nanoscale topography (PAINT)! ${ }^{[10}$ We form a DPPC [di(16:0)PC (phosphatidylcholine), Figure S8 bilayer on coverglass and use RoSE-O to analyze images of blinking NR molecules and extract their positions and orientations. First, we measure NR within a DPPC SLB using both $x y$ Pol and raPol, excited by elliptically polarized epi (Figure 2 c) and single polarization total internal reflection fluorescence (TIRF, Figure 2p) illumination. Interestingly, NR's polar orientation $\theta$ changes significantly with illumination polarization, from a median of $\tilde{\theta}_{\text {epi,raPol }}=80.4^{\circ}$ to $\tilde{\theta}_{\text {TIRF }, \text { raPol }}=41.6^{\circ}$, implying that NR's emission dipole orientation is correlated with the orientation of its absorption dipole moment within a DPPC SLB. To confirm, we changed the laser beam tilt to $\alpha=25^{\circ}$ and $\alpha=45^{\circ}$ relative to the optical axis (Figure 2 $\mathrm{d}, \mathrm{e})$. As the $z$-polarized electric field in the excitation beam increased, the observed NR polar angles systematically decreased $\left(\tilde{\theta}_{\alpha=25^{\circ}}=74.4^{\circ}, \tilde{\theta}_{\alpha=45^{\circ}}=57.7^{\circ}\right)$. The change in polar angle is obvious within the raPol images at each illumination angle $\alpha$; fluorescence photons become increasingly radially polarized as $\alpha$ increases [Figure 2(i)]. These trends were consistently observed across $\sim 35 \mathrm{k}$ localizations and 12 fields of view. 
a
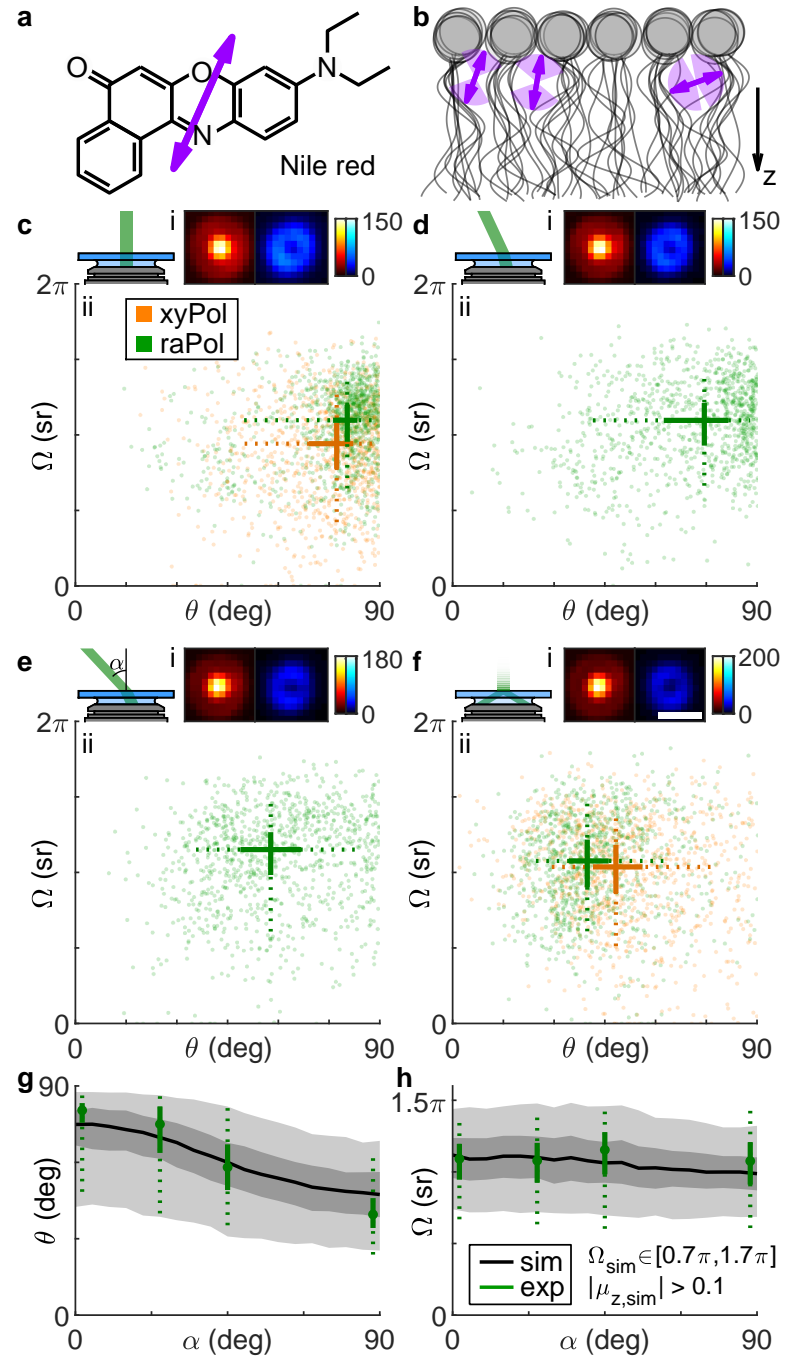

Figure 2: Rotational diffusion of (a) Nile red (NR) within (b) DPPC supported lipid bilayers (SLBs). Arrows represent the direction of the transition dipole moment $\mu$. (c-f) (i) Average raPol images and (ii) orientation and wobble measurements of $\mathrm{NR}$ as a function of illumination tilt angle $\alpha$ : (c) $0^{\circ}$, (d) $25^{\circ}$, (e) $45^{\circ}$, and (f) total internal reflection fluorescence (TIRF). Measurements were collected using both (orange) $x y \mathrm{Pol}$ and (green) raPol imaging. Crosses represent the median of the distribution, while solid lines represent the 33rd and 67th percentiles, and dotted lines represent the 10th and 90th percentiles. (g,h) Quantifying the effect of illumination tilt angles $\alpha$ on measurements of (f) polar angle $\theta$ and $(\mathrm{h})$ wobble $\Omega$. Green: experimental measurements. Dots represent the median, solid lines represent 33rd and 67th percentile, and dotted lines represent the 10th and 90th percentile. Black and gray: simulated orientation measurements of rotationally diffusing SMs, assuming an excited-state lifetime of $4.6 \mathrm{~ns}$, rotational diffusion coefficient of $0.035 \mathrm{rad}^{2} / \mathrm{ns}$, and constrained diffusion within a hard-edged cone of solid angle $\Omega_{\text {sim }} \in[0.7 \pi, 1.7 \pi]$ sr. Lines, dark and light gray areas represent the median, 33rd/67th, and 10th/90th percentiles, respectively. Colorbar: photons; scalebar: $500 \mathrm{~nm}$.

To explain how the polarization of the illumination laser strongly affects the observed NR orientations, we used Monte Carlo simulations to model light absorp- tion, rotational diffusion,, $42[43$ and photon emission of NR within a DPPC SLB, using reported values for its excitedstate lifetime (4.6 ns) and rotational diffusion coefficient $\left(0.035 \mathrm{rad}^{2} / \mathrm{ns}\right) .44$ These simulations generate images of diffusing SMs (SI Section 4.1 and Figure S9), and we use RoSE-O to obtain measurements of their average orientation $\left(\theta_{\text {sim }}, \phi_{\text {sim }}\right)$ and rotational wobble $\Omega_{\text {sim }}$ (Figure $2 \mathrm{~g}, \mathrm{~h}$ ). By simulating many possible models of rotational diffusion (Figure S10, we find that the best match to experimental measurements is produced when each NR molecule has a random average orientation $\boldsymbol{\mu}_{\text {sim }}$ uniformly distributed within the domain $\left|\mu_{z, \operatorname{sim}}\right|>0.1$. In addition, each SM has a random wobble within a hard-edged cone of solid angle $\Omega_{\text {sim }}$ uniformly distributed between $0.7 \pi$ and $1.7 \pi$; a molecule can freely explore all possible orientations within the aforementioned cone. With these parameters, the simulated orientation measurements match both the experimental median and variation in NR orientations reasonably well ( $7.8 \%$ average error in median $\tilde{\theta}, 5.0 \%$ average error in median $\tilde{\Omega})$.

Based on our best-fit model of rotational diffusion, we hypothesize that during a fluorescence "flash," binding sites within the SLB constrain NR molecules such that their dipoles are oriented in all directions except parallel to the membrane (i.e., $\left.\theta \in\left[0^{\circ}, 84^{\circ}\right]\right)$. Given the angle between NR's dipole moment and its structure (Figure 2a), there is thus a $\sim 54^{\circ}$ tilt between the long axis of NR and the membrane normal. 38 Further, steric effects allow NR to explore an intermediate range of orientations (median $\tilde{\Omega}=1.11 \pi$ sr in our experiments irrespective of illumination tilt $\alpha$ ) but prevent it from rotating freely (Figure 2p). Thus, we believe NR binds near the glycerol backbone $e^{44}$ or to kinked acyl chains $\underline{4749}$ of DPPC that are stable on the order of our camera exposure time (100 ms).

We next explore raPol's ability to detect chol-induced ordering dynamics within DPPC SLBs. ${ }^{31}$ Cholesterol is loaded into the bilayer using two successive $\mathrm{M} \beta \mathrm{CD}$-chol treatments (40 $\mu \mathrm{M}$ for 5 minutes, then $80 \mu \mathrm{M}$ for 5 minutes, SI Section 3.2, and we again measured NR orientations using $x y$ Pol and raPol under epi (Figure 3 a) and TIRF excitation (Figure 3b). We observe two distinct "clusters" of NR orientations under both illumination conditions; the first is oriented nearly perpendicular to the SLB (median $\tilde{\theta}_{\text {TIRF }, \text { raPol }, 1}=16.5^{\circ}$ ), which is consistent with previous observations. $\frac{31}{11}$ The polar angles of localizations within this cluster do not change with illumination polarization, except that number of localizations when using epi illumination is $15 \%$ of that using TIRF. Both $x y$ Pol and raPol detect a decrease in rotational wobble (median $\tilde{\Omega}_{\mathrm{TIRF}, \mathrm{raPol}, 1}=0.93 \mathrm{sr}$ ) when chol is present. These data suggest that this population of NR is tightly confined within a small range of orientations during its fluorescence lifetime, which is consistent with our intuition of increased crowding within cholcondensed SLBs.

However, we note a significant discrepancy between $x y \mathrm{Pol}$ and raPol measurements of the second cluster. When using raPol, this other population of NR exhibits similar orientations (median $\tilde{\theta}_{\mathrm{TIRF}, \mathrm{raPol}, 2}=44.5^{\circ}, \tilde{\Omega}_{\mathrm{TIRF}, \mathrm{raPol}, 2}=3.47 \mathrm{sr}$ ) to that of NR within the DPPC-only SLB (Figure 2a, $\tilde{\theta}_{\mathrm{TIRF}, \mathrm{raPol}}=41.6^{\circ}, \tilde{\Omega}_{\mathrm{TIRF}, \mathrm{raPol}}=3.48 \mathrm{sr}$ ). However, there is no clearly resolved second cluster in the $x y$ Pol measurements, just a long tail in the $\theta-\Omega$ distribution [Figure $[\mathrm{S} 13 \mathrm{a}(\mathrm{i})]$. Compared to raPol imaging (Figure 1, f and Table [S3, $x y$ Pol's degraded orientation sensitivity makes it difficult to resolve these two NR orientation behaviors within DPPC-chol SLBs. 
bioRxiv preprint doi: https://doi.org/10.1101/2021.10.19.465033; this version posted December 29, 2021. The copyright holder for this preprint (which was not certified by peer review) is the author/funder, who has granted bioRxiv a license to display the preprint in perpetuity. It is made available under aCC-BY-ND 4.0 International license.

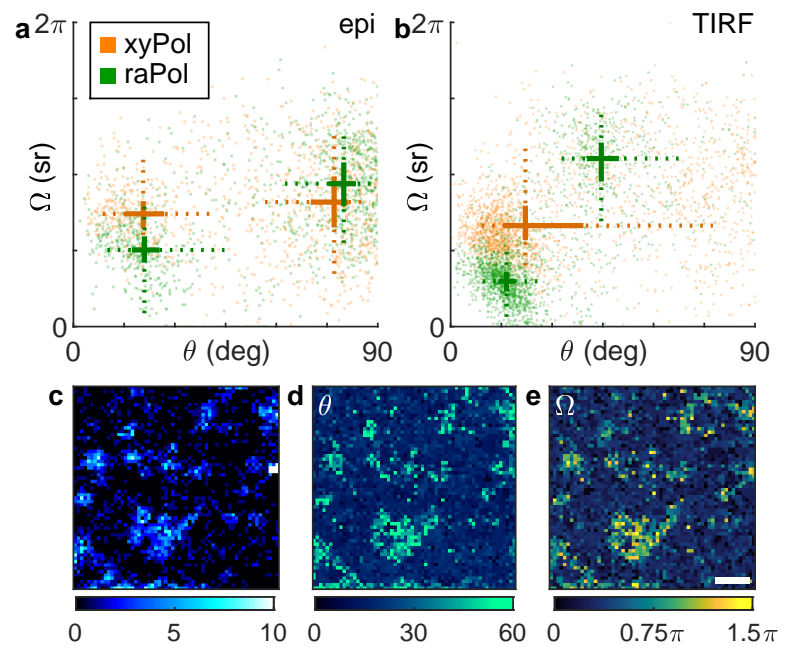

Figure 3: Orientation distribution of NR molecules within DPPC SLBs after cholesterol-loaded methyl- $\beta$-cyclodextrin (MßCD-chol) treatment excited by (a) epi and (b) TIRF illumination and measured using (orange) $x y \mathrm{Pol}$ and (green) raPol. Crosses represent the median of the distribution, while solid lines represent the $33 \mathrm{rd}$ and 67 th percentiles, and dotted lines represent the 10th and 90th percentiles. A kmeans clustering $(k=2)$ is used to separate the distribution into two populations. (c-e) Identifying cholesterol-rich regions within the SLB using raPol and TIRF illumination. (c) SMLM image of NR in the azimuthally polarized imaging channel. (d-e) SMOLM images of (d) median polar angle $\theta$ and (e) median wobble cone angle $\Omega$ of NR localizations in each bin. Colorbars: (c) localizations per bin, (d) polar angle (deg), (e) wobble solid angle (sr). Bin size: $100 \times 100 \mathrm{~nm}^{2}$. Scale bar: $1 \mu \mathrm{m}$.

These two distinct orientation subpopulations raise the question: are these NR binding behaviors uniformly dispersed throughout the SLB, or has chol partitioned the SLB into micro- or nanodomains? We first use ThunderSTORM to localize NR blinking events in the azimuthally polarized channel. Since NR within ordered DPPC-chol SLBs is nearly fixed and perpendicular to the membrane (Figure $3 \mathrm{~b}$ ), azimuthally polarized fluorescence only originates from regions of the SLB that are depleted of cholesterol. We observe an obvious distinction between darker cholesterol-rich regions vs. localization hotspots without cholesterol (Figure 35). Analyzing images from both raPol channels using RoSE-O, we are able to construct SMOLM orientation $\theta$ (Figure 3p) and wobble $\Omega$ (Figure 3p) images, where chol-depleted nanodomains are easy to distinguish due to their large polar and wobble angles. These regions are difficult to detect in standard SMLM reconstructions since NR's localization density is mostly uniform everywhere (Figure S13 d). Interestingly, $x y \mathrm{Pol}$ is able to distinguish between chol-rich and poor regions of the SLB using NR polar angles $\theta$ (Figure S13p), but its map of NR wobble $\Omega$ is extremely noisy (Figure S13.).

With increasing chol in the SLB, we also observed remarkable changes in the residence time of many NR molecules. Here, we track the 2D positions and 3D orientations simultaneously of diffusing NR molecules over multiple frames (see Movie S1 for typical images of NR and Figure S16 $\mathrm{d}$ for the distribution of NR binding times). Within the DPPC-only $\mathrm{SLB}$, one molecule is translationally fixed, but rotates along the polar direction; the brightness ratio between the raPol
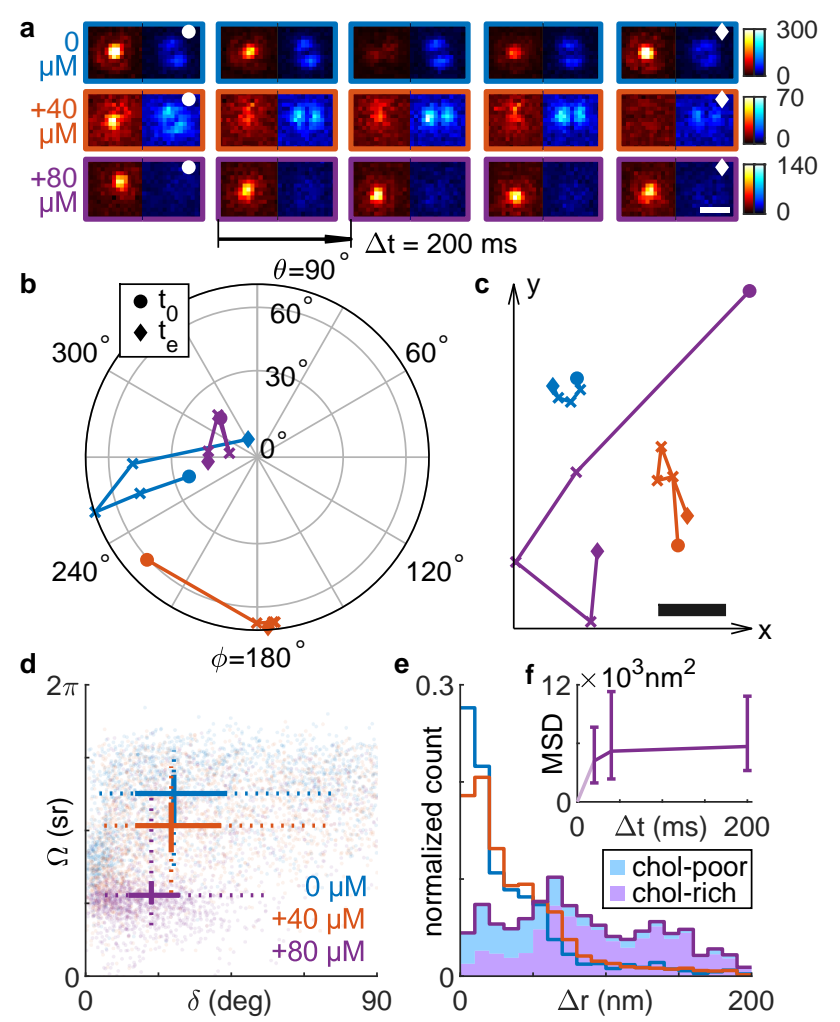

Figure 4: Rotational and translational dynamics of NR within SLBs before and after successive MBCD-chol treatments. Blue: before $\mathrm{M} \beta \mathrm{CD}$-chol treatment; orange: after $40 \mu \mathrm{M}$ treatment for 5 minutes; purple: after $80 \mu \mathrm{M}$ treatment for 5 minutes. (a) Raw raPol images of three SMs undergoing diffusion before and after each treatment. Scale bar: $500 \mathrm{~nm}$; colorbar: photons. (b,c) SM trajectories exhibiting (b) rotational and (c) translational diffusion corresponding to images in (a). Circles and diamonds represent the first $\left(t_{0}\right)$ and last frame $\left(t_{e}\right)$, respectively. The relative position between the three trajectories in (c) is arbitrary. Scale bar: $50 \mathrm{~nm}$. (d) Average angular displacement $\bar{\delta}$ vs. average wobble $\Omega$ for each NR trajectory. Solid lines represent the 33rd and 67th percentiles, and dotted lines represent the 10th and 90th percentiles of each distribution. Intersections represent the median. (e) Measured lateral displacements $\Delta r$ accumulated over all NR trajectories. Shaded area (purple) represents localizations within chol-rich regions of the SLB, as measured by the NR polar angle $\theta$ and wobble $\Omega$. (f) Mean squared displacement (MSD, $\mathrm{nm}^{2}$ ) as a function of time lag $\Delta t$. Errorbars represent the $33 \mathrm{rd} / 67$ th percentile. All data here are from blinking events lasting at least two consecutive frames $[200 \mathrm{~ms}$ for (a-e)].

imaging channels changes over time (Figure 4 a-c, blue). After a single application of M CD-chol, another SM exhibits more translational diffusion and rotates along the azimuthal direction; the double-spot PSF in the azimuthally polarized channel rotates dramatically over time (Figure $4 \mathrm{~A}$-c, orange). After the final treatment, another NR molecule moves a large distance in $x$ and $y$ but remains $z$-oriented throughout its entire trajectory (Figure 4 a-c, purple). Additional representative SM trajectories are shown in Figure S14.

To quantify rotational diffusion between frames, we compute the angular displacement (i.e., rotation) of single NR 
as

$$
\delta_{i}=\cos ^{-1}\left(\boldsymbol{\mu}_{i+1} \cdot \boldsymbol{\mu}_{i}\right)
$$

which represents an angle between two adjacent orientation measurements $\boldsymbol{\mu}_{i}$ and $\boldsymbol{\mu}_{i+1}$ within an SM trajectory. This slow-scale displacement remains unchanged after a first application of $\mathrm{M} \beta \mathrm{CD}$-chol $\left[27.3^{\circ} \pm 26.5^{\circ}\right.$ (median \pm std. dev.) before and $26.4^{\circ} \pm 25.7^{\circ}$ after treatment, respectively, Figure $4 \mathrm{~d}$ ]. Only after a second higher-dose incubation of $\mathrm{M} \beta \mathrm{CD}$-chol does the average SM rotation $\bar{\delta}$ decrease to $20.3^{\circ} \pm 20.2^{\circ}$. In contrast, fast-scale intraframe SM wobbling $\Omega$ decreases successively after each treatment (Figure 4 $\mathrm{d}$ ), from $3.93 \pm 0.96$ sr before treatment to $3.24 \pm 1.05 \mathrm{sr}$ after a $40 \mu \mathrm{M}$ incubation for $5 \mathrm{~min}$., and finally to $1.74 \pm 1.06$ sr after a $80 \mu \mathrm{M}$ treatment.

Even though NR molecules become more rotationally constrained as chol concentration increases, they curiously exhibit successively larger translational motions. The lateral displacement, given by

$$
\Delta r_{i}=\left\|\boldsymbol{r}_{i+1}-\boldsymbol{r}_{i}\right\|
$$

is $20.8 \pm 37.4 \mathrm{~nm}$ (median \pm std. dev., $200 \mathrm{~ms}$ time lag between the $i^{\text {th }}$ and $(i+1)^{\text {th }}$ frame) averaged across all NR localizations before adding M $\beta C D$-chol (Figure 4 $)$. After a $40 \mu \mathrm{M}$ treatment, the average displacement becomes $28.2 \pm 37.5 \mathrm{~nm}$ and grows to $75.5 \pm 50.3 \mathrm{~nm}$ after a $80 \mu \mathrm{M}$ treatment. We gain additional insight into these behaviors by using the polar orientation $\theta$ and wobble $\Omega$ of each localization to separate molecules exploring chol-rich regions of the SLB from those exploring chol-poor ones (using $k$ means classification, see SI Section 4.3. We find that the largest lateral displacements overwhelmingly occur when NR is within chol-rich domains $[\Delta r=44.4 \pm 44.1 \mathrm{~nm}$ in cholpoor vs. $\Delta r=91.5 \pm 47.1 \mathrm{~nm}$ in chol-rich domains, Figure S15.(iii)]. In fact, the proportion of all large lateral displacements $(\Delta r>62.4 \mathrm{~nm}=3 \times$ the median of $\Delta r$ in DPPC SLBs) that originate from chol-rich regions is more than $84.8 \%$. Overall, however, the mean squared displacement (MSD, SI Section 4.2 of all NR trajectories measured after the $80 \mu \mathrm{M}$ treatment scales sublinearly with the time lag $\Delta t$ (Figure 4 f); the MSDs are $(4.2 \pm 13.2) \times 10^{3} \mathrm{~nm}^{2}$, $(5.2 \pm 17.0) \times 10^{3} \mathrm{~nm}^{2}$, and $(5.7 \pm 9.6) \times 10^{3} \mathrm{~nm}^{2}$ for $\Delta t=20 \mathrm{~ms}, 40 \mathrm{~ms}$ and $200 \mathrm{~ms}$, respectively. Thus, NR diffusion is inhomogeneous and largely confined, aside from infrequent jumps, throughout the SLB.

In summary, we design and implement a new PSF, the radially and azimuthally polarized ( $\mathrm{raPol}$ ) standard PSF, for SMOLM that only requires a commercially available waveplate and polarizing beamplitter to be added to a standard epifluorescence microscope. The PSF is significantly more compact than other engineered PSFs. Despite its compactness, raPol's orientation precision is $30.6 \%$ better on average compared to $x y$ Pol. In addition, since $z$-oriented dipoles produce radially polarized light, raPol can directly detect the presence of out-of-plane molecules via a simple quantification of the brightness ratio between its two detection channels (Figure 3.). Thus, it achieves excellent sensitivity for measuring the orientation of a fluorescent molecule..$^{25 \mid 26}$

This combination of superior detection rate, excellent orientation measurement precision, and strong localization precision enables raPol to measure the rotational and translational dynamics of SMs with exquisite detail. In particular, raPol is able to distinguish between depolarization, i.e., fluorescence polarization that is not parallel to the excitation polarization, caused by an immobile tilted SM from an SM exhibiting large rotational diffusion-cases that are difficult to distinguish using time-resolved fluorescence anisotropy. ${ }^{50 \mid 51}$ In addition, tracking the position and orientation of many SMs simultaneously within a field of view allows raPol to map dynamics within the membrane more expediently than point-scanning techniques like stimulated emission depletion (STED) FCS ${ }^{[52153]}$ and polarized FCS, while also offering higher spatial resolution than ensemble measurements. ${ }^{[55]}$ Further, since detection rate and estimation performance are critically important for quantitative imaging, raPol overcomes limitations of orientation-sensing PSFs, e.g., the Tri-spot and Duo-spot PSFs, ${ }^{31}$ which require more photons and longer exposure times to achieve good detection performance; combining raPol with varying illumination polarizations measures NR dynamics with superior accuracy.

Our tracking of NR orientation and wobble within the membrane suggests that it binds near the glycerol backbone or to the kinked lipid tails of DPPC; due to the relative angle between NR's transition dipole and its long axis (Figure 2 ), these binding pockets are unlikely to be normal to the membrane. One possible way to corroborate our observations is to directly label the acyl chains of DPPC at various positions with bifunctional dyes ${ }^{56 / 57}$ and compare their orientational dynamics to that of NR; while outside the scope of this work, these correlative studies would provide powerful insights into lipid conformational dynamics. ${ }^{[4][4]}$ Moreover, the binding pockets can likely be compressed by condensing the acyl chains using cholesterol. ${ }^{31 \mid 58}$ After a first dose of $\mathrm{M} \beta \mathrm{CD}$-chol, the average cholesterol concentration within the membrane is still relatively low (Figure S12p), but measuring the decreasing wobble $\Omega$ reveals the changing chemical composition of the SLB (Figure $4 \mathrm{~d}$ ).

After a second treatment, we find that NR demonstrates a curious combination of diffusive behaviors: strongly confined rotational motions but dramatically larger translational jumps between frames. The distribution of lateral displacements $\Delta r$ (Figure 4 , purple) is non-exponential and broad. NR also largely favors jumping within and between domains of similar chemical composition. We observed 16 times more jumping events between chol-rich regions versus from rich to poor; similarly, once within a chol-poor domain, NR jumped between chol-poor regions at 7.4 times the rate of jumping to chol-rich domains (SI Section 4.3). We surmise that the M $\beta C D$-chol treatments dissolve the SLB in a highly nonuniform manner, causing NR to exhibit "jump diffusion" between regions of intact membrane. NR molecules repeatedly diffuse across damaged portions of the bilayer with widely varying jump distances $\Delta r$ to bind transiently with other chol-rich islands, where their orientations are highly confined upon binding. Thus, NR's rotational and translational dynamics, as observed by raPol SMOLM, support previous observations of cyclodextrin molecules damaging lipid membranes by carrying away lipid molecules after depositing cholesterol. ${ }^{5960}$ This insight into M $\beta C D$-chol activity on the SLB is not detectable by conventional SMLM (Figure S13, e).

Looking ahead, we anticipate that the ease of implementing raPol will enable SMOLM to be adopted more widely by the nanoscience community. For example, raPol can be adapted to map nanodomain partitioning, the saturation of acyl chains, and temperature variations within SLBs; while these phenomena have been studied by tracking SM translational dynamics, ${ }^{61] 63}$ we hypothesize that simultaneous measurements of translational and rotational diffusion 
bioRxiv preprint doi: https://doi.org/10.1101/2021.10.19.465033; this version posted December 29, 2021. The copyright holder for this preprint (which was not certified by peer review) is the author/funder, who has granted bioRxiv a license to display the preprint in perpetuity. It is made available under aCC-BY-ND 4.0 International license.

will enable more sensitive experiments when these effects are more subtle. Such studies would be aided by improvements in image analysis algorithms to increase their automation, robustness, and speed. We look forward to continued developments in SM orientation-localization microscopy for elucidating a more complete picture of chemical dynamics at the nanoscale.

Acknowledgement This work is supported by the National Science Foundation under grant number ECCS1653777 and by the National Institute of General Medical Sciences of the National Institutes of Health under grant number R35GM124858.

\section{Supporting Information Available}

Imaging system schematic and alignment and calibration procedures; characterization of raPol's detection and estimation performance; additional NR orientation-localization analysis; additional references. $\frac{6465}{6}$ The data underlying this study are openly available in OSF at https://osf.io/ 64bfv/?view_only=3a2cbdc3fd20467486f3574d54ecc7f6 and from the corresponding author upon reasonable request.

\section{References}

(1) Engelhardt, J.; Keller, J.; Hoyer, P.; Reuss, M.; Staudt, T.; Hell, S. W. Molecular Orientation Affects Localization Accuracy in Superresolution FarField Fluorescence Microscopy. Nano Letters 2011, 11, 209-213.

(2) Backlund, M. P.; Lew, M. D.; Backer, A. S.; Sahl, S. J.; Grover, G.; Agrawal, A.; Piestun, R.; Moerner, W. E. Simultaneous, accurate measurement of the 3D position and orientation of single molecules. Proceedings of the National Academy of Sciences 2012, 109, 1908719092.

(3) Lew, M. D.; Moerner, W. E. Azimuthal Polarization Filtering for Accurate, Precise, and Robust SingleMolecule Localization Microscopy. Nano Letters 2014, 14, 6407-6413.

(4) Backlund, M. P.; Lew, M. D.; Backer, A. S.; Sahl, S. J.; Moerner, W. E. The Role of Molecular Dipole Orientation in Single-Molecule Fluorescence Microscopy and Implications for Super-Resolution Imaging. ChemPhysChem 2014, 15, 587-599.

(5) Backlund, M. P.; Arbabi, A.; Petrov, P. N.; Arbabi, E.; Saurabh, S.; Faraon, A.; Moerner, W. E. Removing orientation-induced localization biases in singlemolecule microscopy using a broadband metasurface mask. Nature Photonics 2016, 10, 459-462.

(6) Hulleman, C. N.; Thorsen, R. Ø.; Kim, E.; Dekker, C.; Stallinga, S.; Rieger, B. Simultaneous orientation and 3D localization microscopy with a Vortex point spread function. Nature Communications 2021, 12, 5934.

(7) Hess, S. T.; Girirajan, T. P.; Mason, M. D. Ultra-High Resolution Imaging by Fluorescence Photoactivation Localization Microscopy. Biophysical Journal 2006, 91, 4258-4272.
(8) Rust, M. J.; Bates, M.; Zhuang, X. Sub-diffractionlimit imaging by stochastic optical reconstruction microscopy (STORM). Nature Methods 2006, 3, 793796 .

(9) Betzig, E.; Patterson, G. H.; Sougrat, R.; Lindwasser, O. W.; Olenych, S.; Bonifacino, J. S.; Davidson, M. W.; Lippincott-Schwartz, J.; Hess, H. F. Imaging Intracellular Fluorescent Proteins at Nanometer Resolution. Science 2006, 313, 1642-1645.

(10) Sharonov, A.; Hochstrasser, R. M. Wide-field subdiffraction imaging by accumulated binding of diffusing probes. Proceedings of the National Academy of Sciences 2006, 103, 18911-18916.

(11) Shaban, H. A.; Valades-Cruz, C. A.; Savatier, J.; Brasselet, S. Polarized super-resolution structural imaging inside amyloid fibrils using Thioflavine T. Scientific Reports 2017, 7, 12482.

(12) Ding, T.; Wu, T.; Mazidi, H.; Zhang, O.; Lew, M. D. Single-molecule orientation localization microscopy for resolving structural heterogeneities between amyloid fibrils. Optica 2020, 7, 602-607.

(13) Ding, T.; Lew, M. D. Single-Molecule Localization Microscopy of 3D Orientation and Anisotropic Wobble Using a Polarized Vortex Point Spread Function. The Journal of Physical Chemistry B 2021, 125, 1271812729 .

(14) Ha, T.; Enderle, T.; Chemla, D. S.; Selvin, P. R.; Weiss, S. Single Molecule Dynamics Studied by Polarization Modulation. Physical Review Letters 1996, 77, 3979-3982.

(15) Ha, T.; Glass, J.; Enderle, T.; Chemla, D. S.; Weiss, S. Hindered Rotational Diffusion and Rotational Jumps of Single Molecules. Physical Review Letters 1998, 80, 2093-2096.

(16) Backer, A. S.; Lee, M. Y.; Moerner, W. E. Enhanced DNA imaging using super-resolution microscopy and simultaneous single-molecule orientation measurements. Optica 2016, 3, 659-666.

(17) Backer, A. S.; Biebricher, A. S.; King, G. A.; Wuite, G. J. L.; Heller, I.; Peterman, E. J. G. Single-molecule polarization microscopy of DNA intercalators sheds light on the structure of S-DNA. Science Advances 2019, 5, eaav1083.

(18) Mazidi, H.; King, E. S.; Zhang, O.; Nehorai, A.; Lew, M. D. Dense Super-Resolution Imaging of Molecular Orientation Via Joint Sparse Basis Deconvolution and Spatial Pooling. 2019 IEEE 16th International Symposium on Biomedical Imaging (ISBI 2019). 2019; pp 325-329.

(19) Valades Cruz, C. A.; Shaban, H. A.; Kress, A.; Bertaux, N.; Monneret, S.; Mavrakis, M.; Savatier, J.; Brasselet, S. Quantitative nanoscale imaging of orientational order in biological filaments by polarized superresolution microscopy. Proceedings of the National Academy of Sciences 2016, 113, E820-E828. 
(20) Curcio, V.; Alemán-Castañeda, L. A.; Brown, T. G.; Brasselet, S.; Alonso, M. A. Birefringent Fourier filtering for single molecule coordinate and height superresolution imaging with dithering and orientation. $\mathrm{Na}$ ture Communications 2020, 11, 5307.

(21) Rimoli, C. V.; Cruz, C. A. V.; Curcio, V.; Mavrakis, M.; Brasselet, S. 4polar-STORM polarized super-resolution imaging of actin filament organization in cells. bioRxiv 2021, doi.org/10.1101/2021.03.17.435879.

(22) Zhang, O.; Lu, J.; Ding, T.; Lew, M. D. Imaging the three-dimensional orientation and rotational mobility of fluorescent emitters using the Tri-spot point spread function. Applied Physics Letters 2018, 113, 031103.

(23) Opatovski, N.; Shalev Ezra, Y.; Weiss, L. E.; Ferdman, B.; Orange-Kedem, R.; Shechtman, Y. Multiplexed PSF Engineering for Three-Dimensional Multicolor Particle Tracking. Nano Letters 2021, 21, 58885895 .

(24) Zhang, O.; Lew, M. D. Fundamental Limits on Measuring the Rotational Constraint of Single Molecules Using Fluorescence Microscopy. Physical Review Letters 2019, 122, 198301.

(25) Zhang, O.; Lew, M. D. Quantum limits for precisely estimating the orientation and wobble of dipole emitters. Physical Review Research 2020, 2, 033114.

(26) Zhang, O.; Lew, M. D. Single-molecule orientation localization microscopy I: fundamental limits. Journal of the Optical Society of America A 2021, 38, 277.

(27) Backlund, M. P.; Shechtman, Y.; Walsworth, R. L. Fundamental Precision Bounds for Three-Dimensional Optical Localization Microscopy with Poisson Statistics. Physical Review Letters 2018, 121, 023904.

(28) Tsang, M. Quantum limit to subdiffraction incoherent optical imaging. Physical Review A 2019, 99, 012305.

(29) Mortensen, K. I.; Churchman, L. S.; Spudich, J. A.; Flyvbjerg, H. Optimized localization analysis for single-molecule tracking and super-resolution microscopy. Nature Methods 2010, 7, 377-381.

(30) Zhang, O.; Lew, M. D. Single-molecule orientation localization microscopy II: a performance comparison. Journal of the Optical Society of America A 2021, 38, 288.

(31) Lu, J.; Mazidi, H.; Ding, T.; Zhang, O.; Lew, M. D. Single-Molecule 3D Orientation Imaging Reveals Nanoscale Compositional Heterogeneity in Lipid Membranes. Angewandte Chemie International Edition 2020, 59, 17572-17579.

(32) Lew, M. D.; Backlund, M. P.; Moerner, W. E. Rotational Mobility of Single Molecules Affects Localization Accuracy in Super-Resolution Fluorescence Microscopy. Nano Letters 2013, 13, 3967-3972.
(33) Stallinga, S. Effect of rotational diffusion in an orientational potential well on the point spread function of electric dipole emitters. Journal of the Optical Society of America A 2015, 32, 213.

(34) Chandler, T.; Shroff, H.; Oldenbourg, R.; La Rivière, P. Spatio-angular fluorescence microscopy III Constrained angular diffusion, polarized excitation, and high-NA imaging. Journal of the Optical Society of America A 2020, 37, 1465.

(35) Sheppard, C. J.; Wilson, T. The image of a single point in microscopes of large numerical aperture. Proceedings of the Royal Society of London. A. Mathematical and Physical Sciences 1982, 379, 145-158.

(36) Fourkas, J. T. Rapid determination of the threedimensional orientation of single molecules. Optics Letters 2001, 26, 211.

(37) Moon, T. K.; Stirling, W. C. Mathematical Methods and Algorithms for Signal Processing; Prentice Hall, New Jersey, 2000.

(38) Blanchard, G. J. Picosecond spectroscopic measurement of a solvent dependent change of rotational diffusion rotor shape. The Journal of Chemical Physics 1987, 87, 6802-6808.

(39) Dutt, G. B.; Doraiswamy, S.; Periasamy, N.; Venkataraman, B. Rotational reorientation dynamics of polar dye molecular probes by picosecond laser spectroscopic technique. The Journal of Chemical Physics 1990, 93, 8498-8513.

(40) Kawski, A.; Bojarski, P.; Kukliński, B. Estimation of ground- and excited-state dipole moments of Nile Red dye from solvatochromic effect on absorption and fluorescence spectra. Chemical Physics Letters 2008, 463, 410-412.

(41) Zhang, Z.; Yomo, D.; Gradinaru, C. Choosing the right fluorophore for single-molecule fluorescence studies in a lipid environment. Biochimica et Biophysica Acta (BBA) - Biomembranes 2017, 1859, 1242-1253.

(42) Moosaie, A.; Manhart, M. Direct Monte Carlo simulation of turbulent drag reduction by rigid fibers in a channel flow. Acta Mechanica 2013, 224, 2385-2413.

(43) Novikov, A.; Kuzmin, D.; Ahmadi, O. Random walk methods for Monte Carlo simulations of Brownian diffusion on a sphere. Applied Mathematics and Computation 2020, 364, 124670.

(44) Halder, A.; Saha, B.; Maity, P.; Kumar, G. S.; Sinha, D. K.; Karmakar, S. Lipid chain saturation and the cholesterol in the phospholipid membrane affect the spectroscopic properties of lipophilic dye nile red. Spectrochimica Acta Part A: Molecular and Biomolecular Spectroscopy 2018, 191, 104-110.

(45) Kucherak, O. A.; Oncul, S.; Darwich, Z.; Yushchenko, D. A.; Arntz, Y.; Didier, P.; Mély, Y.; Klymchenko, A. S. Switchable Nile Red-Based Probe for Cholesterol and Lipid Order at the Outer Leaflet of Biomembranes. Journal of the American Chemical Society 2010, 132, 4907-4916. 
(46) Sessa, L.; Concilio, S.; Di Martino, M.; Nardiello, A. M.; Miele, Y.; Rossi, F.; Brunetti, J.; Panunzi, B.; Piotto, S. A selective Nile Red based solvatochromic probe: A study of fluorescence in LUVs and GUVs model membranes. Dyes and Pigments 2021, 196, 109759.

(47) Tu, K.; Klein, M. L.; Tobias, D. J. Constant-Pressure Molecular Dynamics Investigation of Cholesterol Effects in a Dipalmitoylphosphatidylcholine Bilayer. Biophysical Journal 1998, 75, 2147-2156.

(48) Hofsäß, C.; Lindahl, E.; Edholm, O. Molecular Dynamics Simulations of Phospholipid Bilayers with Cholesterol. Biophysical Journal 2003, 84, 2192-2206.

(49) Vanni, S.; Riccardi, L.; Palermo, G.; Vivo, M. D. Structure and Dynamics of the Acyl Chains in the Membrane Trafficking and Enzymatic Processing of Lipids. Accounts of Chemical Research 2019, 52, 3087-3096.

(50) Holmes, A.; Birch, D.; Sanderson, A.; Aloisi, G. Timeresolved fluorescence photophysics of trans-stilbene in a DPPC lipid bilayer: evidence for a free rotation, location within two sites and a pre-liquid crystalline phase transition. Chemical Physics Letters 1997, 266, 309316.

(51) Loura, L. M.; Ramalho, J. P. Location and dynamics of acyl chain NBD-labeled phosphatidylcholine (NBDPC) in DPPC bilayers. A molecular dynamics and time-resolved fluorescence anisotropy study. Biochimica et Biophysica Acta (BBA) - Biomembranes 2007, 1768, 467-478.

(52) Honigmann, A.; Mueller, V.; Ta, H.; Schoenle, A.; Sezgin, E.; Hell, S. W.; Eggeling, C. Scanning STED-FCS reveals spatiotemporal heterogeneity of lipid interaction in the plasma membrane of living cells. Nature Communications 2014, 5, 5412.

(53) Sezgin, E.; Schneider, F.; Galiani, S.; Urbančič, I.; Waithe, D.; Lagerholm, B. C.; Eggeling, C. Measuring nanoscale diffusion dynamics in cellular membranes with super-resolution STED-FCS. Nature Protocols 2019, 14 .

(54) Yamamoto, J.; Matsui, A.; Gan, F.; Oura, M.; Ando, R.; Matsuda, T.; Gong, J. P.; Kinjo, M. Quantitative evaluation of macromolecular crowding environment based on translational and rotational diffusion using polarization dependent fluorescence correlation spectroscopy. Scientific Reports 2021, 11, 10594.

(55) Lamazière, A.; Maniti, O.; Wolf, C.; Lambert, O.; Chassaing, G.; Trugnan, G.; Ayala-Sanmartin, J. Lipid domain separation, bilayer thickening and pearling induced by the cell penetrating peptide penetratin. Biochimica et Biophysica Acta (BBA) - Biomembranes 2010, 1798, 2223-2230.

(56) Griffin, B. A.; Adams, S. R.; Tsien, R. Y. Specific Covalent Labeling of Recombinant Protein Molecules Inside Live Cells. Science 1998, 281, 269-272.
(57) Corrie, J. E. T.; Brandmeier, B. D.; Ferguson, R. E.; Trentham, D. R.; Kendrick-Jones, J.; Hopkins, S. C.; van der Heide, U. A.; Goldman, Y. E.; SabidoDavid, C.; Dale, R. E.; Criddle, S.; Irving, M. Dynamic measurement of myosin light-chain-domain tilt and twist in muscle contraction. Nature 1999, 400, 425-430.

(58) Huang, J.; Feigenson, G. W. A Microscopic Interaction Model of Maximum Solubility of Cholesterol in Lipid Bilayers. Biophysical Journal 1999, 76, 2142-2157.

(59) Huang, Z.; London, E. Effect of Cyclodextrin and Membrane Lipid Structure upon Cyclodextrin-Lipid Interaction. Langmuir 2013, 29, 14631-14638.

(60) Gharib, R.; Fourmentin, S.; Charcosset, C.; GreigeGerges, H. Effect of hydroxypropyl- $\beta$-cyclodextrin on lipid membrane fluidity, stability and freeze-drying of liposomes. Journal of Drug Delivery Science and Technology 2018, 44, 101-107.

(61) Vaz, W. L. C.; Clegg, R. M.; Hallmann, D. Translational diffusion of lipids in liquid crystalline phase phosphatidylcholine multibilayers. A comparison of experiment with theory. Biochemistry 1985, 24, 781-786.

(62) Lindblom, G.; Orädd, G.; Filippov, A. Lipid lateral diffusion in bilayers with phosphatidylcholine, sphingomyelin and cholesterol. Chemistry and Physics of Lipids 2006, 141, 179-184.

(63) Hsieh, C.-L.; Spindler, S.; Ehrig, J.; Sandoghdar, V. Tracking Single Particles on Supported Lipid Membranes: Multimobility Diffusion and Nanoscopic Confinement. The Journal of Physical Chemistry B 2014, 118, 1545-1554.

(64) Mazidi, H.; Lu, J.; Nehorai, A.; Lew, M. D. Minimizing Structural Bias in Single-Molecule Super-Resolution Microscopy. Scientific Reports 2018, 8, 13133.

(65) Ferdman, B.; Nehme, E.; Weiss, L. E.; Orange, R.; Alalouf, O.; Shechtman, Y. VIPR: vectorial implementation of phase retrieval for fast and accurate microscopic pixel-wise pupil estimation. Optics Express 2020, 28, 10179. 
bioRxiv preprint doi: https://doi.org/10.1101/2021.10.19.465033; this version posted December 29, 2021. The copyright holder for this preprint (which was not certified by peer review) is the author/funder, who has granted bioRxiv a license to display the preprint in perpetuity. It is made available under aCC-BY-ND 4.0 International license.

\section{TOC Graphic}

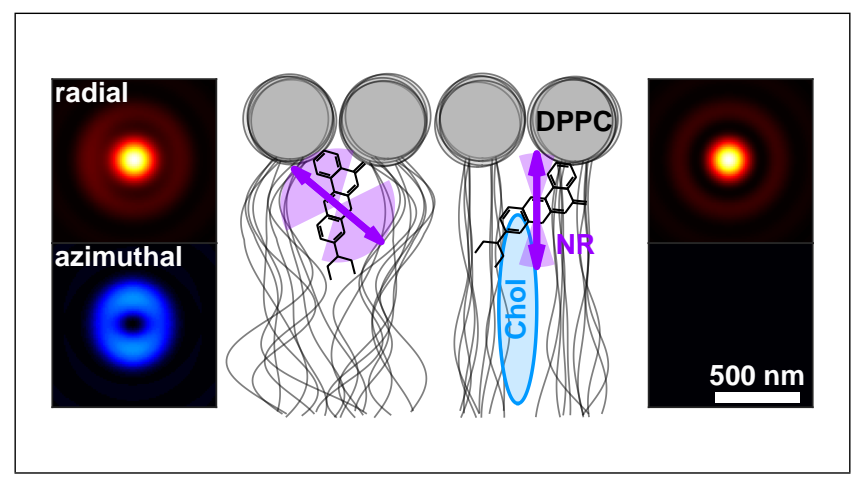

